\title{
THE ZETA FUNCTION OF THE LAPLACIAN ON CERTAIN FRACTALS
}

\author{
GREGORY DERFEL, PETER J. GRABNER, AND FRITZ VOGL
}

\begin{abstract}
We prove that the zeta function $\zeta_{\Delta}$ of the Laplacian $\Delta$ on selfsimilar fractals with spectral decimation admits a meromorphic continuation to the whole complex plane. We characterise the poles, compute their residues, and give expressions for some special values of the zeta function. Furthermore, we discuss the presence of oscillations in the eigenvalue counting function, thereby answering a question posed by J. Kigami and M. Lapidus for this class of fractals.
\end{abstract}

\section{INTRODUCTION}

Diffusion on fractals has been studied extensively as a generalisation of usual Brownian motion on manifolds. After its introduction in the physics literature (cf. [30]) M. Barlow and E. Perkins [3] gave a very detailed study of the properties of the diffusion on the Sierpiński gasket. Later T. Lindstrøm [23] generalised these results to nested fractals. Especially, he derived results on the distribution of the eigenvalues of the Laplacian associated to the diffusion. Here the Laplacian is seen as the infinitesimal generator of Brownian motion. Alternatively, the Laplacian can be obtained as the limit of properly rescaled difference operators on graphs approximating the fractal structure (cf. $[9,14,16])$. Furthermore, the Laplace operator can be obtained via Dirichlet forms (cf. [1]).

There exists a vast literature on properties of the spectrum of the Laplacian. For instance, we refer to $[2,15,16,17,18,21,24,32,34,35,36]$. Especially, it has been proved that the Laplacian on an infinite post-critically-finite (p.c.f.) self-similar fractal has pure point spectrum (cf. [32]).

In the case of a compact self-similar fractal $G$ equipped with the Hausdorff measure $\mathcal{H}$ it is natural to ask for the behaviour of the counting function of the eigenvalues under Dirichlet or Neumann boundary conditions

$$
L(x)=\sum_{\substack{\Delta u=\mu u \\ \mu<x}} 1,
$$

Received by the editors June 23, 2005 and, in revised form, November 4, 2005.

2000 Mathematics Subject Classification. Primary 30B50; Secondary 11M41, 37F10.

Key words and phrases. Dirichlet series, Laplace operator, fractals, spectral decimation, complex dynamics.

The second author was supported by the Austrian Science Fund project S9605. 
the trace of the heat kernel $p_{t}(x, y)$

$$
P(t)=\sum_{-\Delta u=\mu u} e^{-\mu t}=\int_{G} p_{t}(x, x) d \mathcal{H}(x),
$$

or the corresponding Dirichlet generating function

$$
\zeta_{\Delta}(s)=\sum_{-\Delta u=\mu u} \frac{1}{\mu^{s}},
$$

the "zeta function" of the Laplace operator (the eigenvalues are counted with multiplicities in all three sums). This zeta function will be the subject of the present paper.

In the case of the Laplacian on a Riemannian manifold, the meromorphic continuation of $\zeta_{\Delta}$ can be derived directly from the asymptotic expansion of the trace of the heat-kernel (cf. $[27,31])$. In this case it is known that $\zeta_{\Delta}$ admits a meromorphic continuation to the whole complex plane with at most simple poles at $\frac{d}{2}, \frac{d}{2}-1, \ldots, \frac{d}{2}-\left\lfloor\frac{d-1}{2}\right\rfloor$, where $d$ denotes the dimension of the manifold. Furthermore, the residues at these poles have a geometric meaning.

In the case of a fractal the situation is more delicate. The current knowledge on the behaviour of the heat-kernel is not sufficient to provide enough information on its trace to get the meromorphic continuation of $\zeta_{\Delta}$. In the present paper we will present a method for the continuation of $\zeta_{\Delta}$ which is based on an asymptotic study of the solution of Poincaré's functional equation. In Section 3 we prove several results relating the behaviour of the solution to properties of the according Julia set. This approach for the analytic continuation of $\zeta_{\Delta}$ is restricted to a subclass of selfsimilar fractals which exhibit spectral decimation (cf. [30]) with a polynomial map (see Section 2). On the other hand it allows us to attach a zeta function to every polynomial which has a certain behaviour under iteration. Such zeta functions have been introduced in [42]; we give a totally different approach for their meromorphic continuation and are also able to derive expressions for some special values.

In Section 6 we use the properties of the zeta function to confirm a conjecture (cf. [17, p. 105]) about the presence of oscillations in the eigenvalue counting function of the Laplacian for the class of fractals under consideration.

\section{Eigenvalues of the LAPlacian AND SPECTRAL DeCimation}

As it was observed in the case of the Sierpiński gasket and its higher dimensional analogues in $[9,34,35]$, the eigenvalues of the Laplace operator on certain finitely ramified self-similar fractals exhibit the phenomenon of spectral decimation (cf. [9] for the case of higher-dimensional Sierpiński gaskets and [25, 37, 42] for a more general treatment).

Definition 1 (Spectral decimation). The Laplace operator on a p.c.f. self-similar fractal $G$ admits spectral decimation, if there exists a rational function $R$, a finite set $A$ and a constant $\lambda>1$ such that all eigenvalues of $\Delta$ can be written in the form

$$
\lambda^{m} \lim _{n \rightarrow \infty} \lambda^{n} R^{(-n)}(\{w\}), \quad w \in A, m \in \mathbb{N},
$$

where the preimages of $w$ under $n$-fold iteration of $R$ have to be chosen such that the limit exists. Furthermore, the multiplicities of the eigenvalues depend only on $w$ and $m$, and the generating functions of the multiplicities are rational. 
The fact that all eigenvalues of $\Delta$ are negative real implies that the Julia set of $R$ has to be contained in the negative real axis. We will exploit this fact later.

In many cases such as the higher-dimensional Sierpiński gaskets, the rational function $R$ is a polynomial. The method for meromorphic continuation of $\zeta_{\Delta}$ given in Section 5 makes use of this assumption. In a recent paper [42] A. Teplyaev showed under the same assumption that the $\zeta$-function of the Laplacian admits a meromorphic continuation to $\Re s>-\varepsilon$ for some $\varepsilon>0$ depending on properties of the Julia set of the polynomial given by spectral decimation. His method uses ideas similar to those used in [10] for the meromorphic continuation of a Dirichlet series attached to a polynomial. Complementary to the ideas used here, Teplyaev's method carries over to rational functions $R$.

\section{Asymptotic Behaviour of SOLUTIONS OF A FUnCTIONAL EQUATION}

In this section we collect several results on the growth of the solution of the Poincaré functional equation related to polynomials. We note here that Valiron [44, Chapter VII] gave rather general results on the order of growth of the solution of such functional equations. Throughout the paper we will use the notation

$$
\log _{\lambda} z=\frac{\log z}{\log \lambda}
$$

for the logarithm to base $\lambda$. We first present a classical result.

Lemma 1 (Valiron [44]). Let $p(x)=a_{d} x^{d}+\cdots+a_{1} x$ be a polynomial with real coefficients, $d \geq 2$, which satisfies $p(0)=0$ and $p^{\prime}(0)=\lambda>1$. Any formal power series solution $\Phi(z)$ of the functional equation

$$
\Phi(\lambda z)=p(\Phi(z)), \quad \Phi(0)=0, \quad \Phi^{\prime}(0)=1
$$

is an entire function of order

$$
\rho=\frac{\log m}{\log |\lambda|} .
$$

Furthermore, denote $M(r)=\max _{|z| \leq r}|f(z)|$. Then

$$
\log M(r) \sim r^{\rho} Q\left(\frac{\log r}{\log |\lambda|}\right), \quad r \rightarrow \infty,
$$

where $Q$ is a 1-periodic function bounded between two positive constants.

The following theorem gives a more precise description of the growth order of $\Phi$ under assumptions on the behaviour of $p$ under iteration.

Theorem 1. Let $p(x)=a_{d} x^{d}+\cdots+a_{1} x$ be a polynomial with real coefficients, $d \geq 2$, which satisfies $p(0)=0$ and $p^{\prime}(0)=\lambda>1$. Furthermore, suppose that $\mathcal{F}_{\infty}$, the Fatou component of $\infty$ of $p$, contains an angular region of the form

$$
W_{\beta}=\{z \in \mathbb{C} \backslash\{0\}|| \arg z \mid<\beta\}
$$

for some $\beta>0$. Let $\Phi$ be an entire solution of the functional equation (3.1). Then for any $\varepsilon>0$ and any $M>0$ the asymptotic relation

$$
\Phi(z)=\frac{1}{\sqrt[d-1]{a_{d}}} \exp \left(z^{\rho} F\left(\log _{\lambda} z\right)+o\left(|z|^{-M}\right)\right)
$$

holds uniformly for $|\arg z| \leq \beta-\varepsilon$, where $\rho=\log _{\lambda} d$ and $F$ is a periodic holomorphic function of period 1 on the strip $\left\{w \in \mathbb{C}|| \Im w \mid<\frac{\beta}{\log \lambda}\right\}$. The real part of $z^{\rho} F\left(\log _{\lambda} z\right)$ is always positive; $F$ takes real values on the real axis. 
Proof. After Lemma 1 there only remains to prove the assertion about the asymptotic behaviour of $\Phi$.

Since $p$ is a polynomial of degree $d \geq 2$, there exists a real number $R>0$, such that $|z| \geq R \Rightarrow|p(z)| \geq 2|z|$, which implies that $\left(p^{(n)}(z)\right)_{n}$ tends to infinity for $|z|>R$. Fix $\varepsilon>0$. Then by local conformity of $\Phi$ around 0 there exists $r>0$ such that

$$
\Phi(\{z \in \mathbb{C} \backslash\{0\}|| z|\leq r,| \arg z \mid \leq \beta-\varepsilon\}) \subset W_{\beta} .
$$

By definition of the Fatou set the sequence $\left(p^{(n)}(z)\right)_{n}$ is equicontinuous on $W_{\beta}$ and therefore contains a subsequence $\left(p^{\left(n_{k}\right)}(z)\right)_{k}$, which converges to $\infty$ uniformly on

$$
M_{\varepsilon}=\Phi\left(\left\{z \in \mathbb{C} \backslash\{0\}\left|\frac{r}{\lambda} \leq\right| z|\leq r,| \arg z \mid \leq \beta-\varepsilon\right\}\right)
$$

by the fact that $\infty$ is an attracting fixed point of $p$ and by our assumptions on the Fatou component of $\infty$. Thus there exists an $N$ such that $\left|p^{(N)}(z)\right|>R$ for all $z \in M_{\varepsilon}$, which implies that $\left(p^{(n)}(z)\right)_{n}$ converges to $\infty$ uniformly on $M_{\varepsilon}$. Inserting this fact into (3.1) this implies that

$$
\lim _{z \rightarrow \infty}|\Phi(z)|=\infty \text { uniformly for }|\arg z| \leq \beta-\varepsilon .
$$

Furthermore, for any $K>1$ there exists a $C>0$ such that

$$
|\Phi(z)| \geq C|z|^{K} \text { for }|\arg z| \leq \beta-\varepsilon .
$$

Now note that our assumption on the Fatou set implies that there are no zeros of $\Phi$ in $W_{\beta}$. Therefore,

$$
\Psi(z)=\log \Phi(z)
$$

is analytic in $W_{\beta}$ and satisfies the functional equation

$$
\Psi(\lambda z)=d \Psi(z)+\log a_{d}+r(z) \text { with } r(z)=\log \frac{p(\Phi(z))}{a_{d} \Phi(z)^{d}} .
$$

The function $r(z)$ is analytic in

$$
\left\{z \in \mathbb{C}|| z\left|>r_{0},\right| \arg z \mid<\beta\right\}
$$

for some $r_{0}>0$. Setting $\Psi(z)=z^{\rho} \phi(z)-\frac{\log a_{d}}{d-1}$ with $\rho=\log _{\lambda} d$ yields

$$
\phi(\lambda z)=\phi(z)+\frac{r(z)}{d z^{\rho}} .
$$

By our previous knowledge on $\Phi(z)$ for $z \rightarrow \infty$ in $|\arg z|<\beta$ it follows that $r(z)$ tends to 0 faster than any negative power of $|z|$ uniformly in any angular region $|\arg z| \leq \beta-\varepsilon$. Iterating (3.5) yields

$$
\phi\left(\lambda^{n} z\right)=\phi(z)+\frac{1}{z^{\rho}} \sum_{k=0}^{n-1} \frac{r\left(\lambda^{k} z\right)}{d^{k+1}} .
$$

We set

$$
F\left(\log _{\lambda} z\right)=\lim _{n \rightarrow \infty} \phi\left(\lambda^{n} z\right)
$$

which exists by our knowledge on $r(z)$ and (3.6). Clearly, $F(w)$ is periodic with period 1, holomorphic in $|\Im w|<\frac{\beta}{\log \lambda}$ and

$$
\phi(z)=F\left(\log _{\lambda} z\right)-\frac{1}{z^{\rho}} \sum_{k=0}^{\infty} \frac{r\left(\lambda^{k} z\right)}{d^{k+1}}=F\left(\log _{\lambda} z\right)+o\left(|z|^{-M}\right)
$$

for any positive $M$. 
Putting everything together, we obtain

$$
\Psi(z)=z^{\rho} F\left(\log _{\lambda} z\right)-\frac{\log a_{d}}{d-1}+o\left(|z|^{-M}\right),
$$

which implies (3.2). The statement on the real part of $z^{\rho} F\left(\log _{\lambda} z\right)$ follows from $|\Phi(z)| \rightarrow \infty$ for $|z| \rightarrow \infty$ in $W_{\beta}$.

Corollary 2. The limit

$$
\lim _{n \rightarrow \infty} \frac{\log \Phi\left(\lambda^{n} z\right)}{d^{n} z^{\rho}}
$$

exists for all $z \in \Phi^{(-1)}\left(\mathcal{F}_{\infty}\right)$. This limit gives the analytic continuation of $F\left(\log _{\lambda} z\right)$ to $\Phi^{(-1)}\left(\mathcal{F}_{\infty}\right)$.

Proof. The proof of the asymptotic formula for $\Psi(z)$ used only that $\Phi\left(\lambda^{n} z\right) \rightarrow \infty$ for $n \rightarrow \infty$. This relation holds for any point $z \in \Phi^{(-1)}\left(\mathcal{F}_{\infty}\right)$.

Remark 1. Constancy of the periodic function $F$ occurring in the statement of Theorem 1 has been the subject of a series of papers in the context of branching processes $[5,8]$, where it turns out that the constancy of $F$ is implied by the existence of a continuous time extension of the according branching process (cf. [13]). Furthermore, it is also conjectured that the opposite implication is true. Usually, on the real axis the function $F$ exhibits very small oscillations around a mean value, which can only be observed using high precision numerical computations. Therefore, theoretical conditions for the presence of such oscillations are of special interest.

For the purposes of this paper we give the following condition for non-constancy of the periodic function $F$.

Theorem 3. Let $\Phi$ be the solution of the functional equation (3.1). Assume that there exists an angle $\gamma$ such that $\Phi\left(\left\{r e^{i \gamma} \mid r>0\right\}\right)$ intersects the Fatou-component $\mathcal{F}_{\infty}$ as well as the Julia-set $\mathcal{J}_{p}$. Then the periodic function $F$ in (3.2) is not constant.

Proof. We note first that $\mathcal{J}_{p}$ is a compact subset of $\mathbb{C}$, since $p$ is a polynomial (cf. [4]). Assume that $F\left(\log _{\lambda} z\right)=C$. Then by Corollary 2 we have

$$
\lim _{n \rightarrow \infty} \frac{\log \Phi\left(\lambda^{n} z\right)}{d^{n} z^{\rho}}=C
$$

for $z \in \Phi^{(-1)}\left(\mathcal{F}_{\infty}\right)$. For any $r>0$ with $\Phi\left(r e^{i \gamma}\right) \in \mathcal{J}_{p}$ we have $\Phi\left(r \lambda^{n} e^{i \gamma}\right) \in \mathcal{J}_{p}$ for all $n$. Thus by our assumptions, for any $k \in \mathbb{N}$, any $\varepsilon>0$, and any $M>0$ there exists $r>M$, such that $\Phi\left(r e^{i \gamma}\right) \in \mathcal{F}_{\infty}$ and

$$
\inf \left\{\left|\Phi\left(r e^{i \gamma}\right)-z\right| \mid z \in \mathcal{J}_{p}\right\}<\varepsilon
$$

Then we have

$$
\begin{aligned}
& \lim _{n \rightarrow \infty} \frac{\log \Phi\left(\lambda^{n+k} r e^{i \gamma}\right)}{r^{\rho} d^{n+k} e^{i \rho \gamma}}=C, \\
& \lim _{n \rightarrow \infty} \frac{\log p^{(k)}\left(\Phi\left(\lambda^{n} r e^{i \gamma}\right)\right)}{d^{n}\left(p^{(k)}\left(\Phi\left(r e^{i \gamma}\right)\right)\right)^{\rho}}=C .
\end{aligned}
$$

On the other hand

$$
\lim _{n \rightarrow \infty} \frac{\log \Phi\left(\lambda^{n+k} r e^{i \gamma}\right)}{r^{\rho} d^{n+k} e^{i \rho \gamma}}=\lim _{n \rightarrow \infty} \frac{\log p^{(k)}\left(\Phi\left(\lambda^{n} r e^{i \gamma}\right)\right)}{d^{n}\left(p^{(k)}\left(\Phi\left(r e^{i \gamma}\right)\right)\right)^{\rho}} \frac{\left(p^{(k)}\left(\Phi\left(r e^{i \gamma}\right)\right)\right)^{\rho}}{d^{k} r^{\rho} e^{i \gamma \rho}}=C,
$$


since $p^{(k)}\left(\Phi\left(r e^{i \gamma}\right)\right)$ is contained in a bounded set and $r$ can be chosen arbitrarily large. This gives a contradiction.

Corollary 4. Assume that $\mathcal{J}_{p} \subset \mathbb{R}^{-}$and that $\Phi$ is unbounded on $\mathbb{R}^{-}$. Then the periodic function $F$ in Theorem 1 is not constant.

Proof. $\Phi\left(\mathbb{R}^{-}\right)$intersects the Julia set $\mathcal{J}_{p}$, since $\Phi$ attains negative values close to 0 , and $0 \in \mathcal{J}_{p} . \Phi\left(\mathbb{R}^{-}\right)$intersects $\mathcal{F}_{\infty}$, since $\Phi$ takes arbitrarily large values. Then Theorem 3 gives the assertion with $\gamma=\pi$.

Remark 2. The example of $p(x)=x(4+x)$, where $\Phi(z)=4 \sinh ^{2} \frac{1}{2} \sqrt{z}$, shows that the assumption on the unboundedness of $\Phi$ on $\mathbb{R}^{-}$cannot be omitted.

Corollary 5. If $\rho<\frac{1}{2}$, then the periodic function $F$ in Theorem 1 is not constant.

Proof. By [6, Theorem 3.1.5] a function of order $<\frac{1}{2}$ is unbounded on any ray, and so is $\Phi$. Apply Corollary 4 to a ray whose $\Phi$-image intersects the Julia set of $p$ to obtain the assertion.

Corollary 6. If the periodic function $F$ in Theorem 1 is not constant, it has infinitely many non-zero Fourier-coefficients.

Proof. Since $p(0)=0$ and 0 lies on the boundary of $\mathcal{F}_{\infty}$ by the hypotheses of Theorem 1, infinitely many zeros of $\Phi$ lie on the boundary of $\Phi^{(-1)}\left(\mathcal{F}_{\infty}\right)$. From this we get that $F\left(\log _{\lambda} z\right)$ is unbounded when approaching these boundary points of $\Phi^{(-1)}\left(\mathcal{F}_{\infty}\right)$, which implies that $F$ has to have infinitely many non-zero Fouriercoefficients.

\section{Spectral Decimation AND Level Sets of $\Phi$}

By (2.1) all eigenvalues of the Laplacian $\Delta$ can be computed by iterating $p^{(-1)}$ and rescaling. In this section we want to analyse this more precisely. The inverse map of the polynomial function $p$ has $d=\operatorname{deg} p$ branches, which we denote by $q_{1}, \ldots, q_{d}$. We denote by $q_{1}$ the unique branch given by $q_{1}(0)=0$, which also satisfies $q_{1}^{\prime}(0)=\frac{1}{\lambda}$. Given $w \in \mathbb{C}$ the elements of $p^{(-n)}(\{w\})$ are in correspondence to words of length $n$ over the alphabet $\{1, \ldots, d\}$ : associate to $\ell=\left(\ell_{1}, \ldots, \ell_{n}\right) \in$ $\{1, \ldots, d\}^{n}$ the value $z_{\ell}=q_{\ell_{n}} \circ q_{n-1} \circ \cdots \circ q_{\ell_{1}}(w)$. Then $p^{(n)}\left(z_{\ell}\right)=w$ and all elements of $p^{(-n)}(\{w\})$ are of this form.

Let $\boldsymbol{\ell}=\left(\ell_{1}, \ell_{2}, \ldots\right) \in\{1, \ldots, d\}^{\mathbb{N}}$. Then the limit

$$
\lim _{n \rightarrow \infty} \lambda^{n} q_{\ell_{n}} \circ q_{n-1} \circ \cdots \circ q_{\ell_{1}}(w)
$$

exists if and only if there exists $N \in \mathbb{N}$, such that $\ell_{n}=1$ for $n \geq N$. This follows from the fact that $q_{1}$ is the only branch of $p^{(-1)}$ which maps 0 to itself.

Take $z_{n} \in \lambda^{n} p^{(-n)}(\{w\})$. Then define $u_{n} \in \mathbb{C}$ by $\lambda^{-n} z_{n}=\Phi\left(\lambda^{-n} u_{n}\right)$. Then $z_{n}=\lambda^{n} \Phi\left(\lambda^{-n} u_{n}\right)$ and assuming that the $\operatorname{limit}_{n \rightarrow \infty} z_{n}$ exists as discussed above, we have

$$
z=\lim _{n \rightarrow \infty} z_{n}=\lim _{n \rightarrow \infty} \lambda^{n} \Phi\left(\lambda^{-n} u_{n}\right)=\lim _{n \rightarrow \infty} u_{n} .
$$

On the other hand $w=p^{(n)}\left(\Phi\left(\lambda^{-n} u_{n}\right)\right)=\Phi\left(u_{n}\right)$ and therefore $\Phi(z)=w$. Thus the solutions of $\Phi(z)=w$ are exactly the possible limits of sequences $\left(z_{n}\right)$ as above. 
Summing up, we have

Lemma 2. Let $\ell=\left(\ell_{1}, \ell_{2}, \ldots\right) \in\{1, \ldots, d\}^{\mathbb{N}}$ with $\ell_{N}=\ell_{N+1}=\ldots=1$ for some $N \in \mathbb{N}$. Then the limit

$$
z_{\ell}=\lim _{n \rightarrow \infty} \lambda^{n} q_{\ell_{n}} \circ q_{n-1} \circ \cdots \circ q_{\ell_{1}}(w)
$$

exists and satisfies $\Phi\left(z_{\ell}\right)=w$. All solutions of the equation $\Phi(z)=w$ can be obtained as such a limit.

\section{Weierstrass PRODUCtS AND Dirichlet SERIES}

Since the solution $\Phi$ of the functional equation (3.1) is of order $\rho$, we can represent $\Phi_{w}(z)=1-\frac{1}{w} \Phi(z)$ by a Hadamard product (for $w \neq 0$ )

$$
\begin{aligned}
& \Phi_{w}(z)=\exp \left(c_{1}(w) z+\cdots+c_{k}(w) z^{k}\right) \\
& \quad \times \prod_{\ell=1}^{\infty}\left(1+\frac{z}{\mu_{\ell}(w)}\right) \exp \left(-\frac{z}{\mu_{\ell}(w)}+\frac{z^{2}}{2 \mu_{\ell}(w)^{2}}+\cdots+(-1)^{k} \frac{z^{k}}{k \mu_{\ell}(w)^{k}}\right),
\end{aligned}
$$

where $k=\lfloor\rho\rfloor$ denotes the integer part of $\rho$ (cf. [6]). It is clear from the validity of the functional equation (3.1) that $\Phi_{w}$ has infinitely many zeroes even in the case of integer $\rho$.

It follows from Theorem 1 that $\log \Phi_{w}(z)$ has a convergent Taylor series around 0 :

$$
\log \Phi_{w}(z)=\sum_{\ell=1}^{\infty} b_{\ell}(w) z^{\ell} .
$$

Furthermore, assuming that the Julia set of $p$ is a subset of the negative reals and $w<0$ as it is the case for spectral decimation, $\log \Phi_{w}(z)$ is holomorphic in $\{z \in \mathbb{C}|| \arg z \mid<\beta\}$. Computing the Taylor series of $\log \Phi_{w}(z)$ around $z=0$ and comparing coefficients yields $c_{\ell}(w)=b_{\ell}(w)$ for $\ell=1, \ldots, k$.

Let

$$
q_{m}(z, w)=\sum_{\ell=1}^{m} b_{\ell}(w) z^{\ell} \quad \text { for } m \in \mathbb{N}
$$

and consider the Mellin transform of $\log \Phi_{w}(x)-q_{k}(x, w)$,

$$
M_{w}(s)=\int_{0}^{\infty}\left(\log \Phi_{w}(x)-q_{k}(x, w)\right) x^{s-1} d x .
$$

The function $M_{w}(s)$ is holomorphic in the strip $-k-1<\Re s<-\rho$ by general properties of the Mellin transform (cf. [7]). In order to obtain a meromorphic continuation of $M_{w}(s)$ we split the integral in (5.3) into two parts:

$$
\begin{aligned}
& M_{w}^{(1)}(s)=\int_{0}^{1}\left(\log \Phi_{w}(x)-q_{k}(x, w)\right) x^{s-1} d x, \\
& M_{w}^{(2)}(s)=\int_{1}^{\infty}\left(\log \Phi_{w}(x)-q_{k}(x, w)\right) x^{s-1} d x .
\end{aligned}
$$


It is clear that $M_{w}^{(1)}(s)$ is holomorphic in $\Re s>-k-1$. From the expansion (5.2) of $\log \Phi_{w}(x)$ we immediately get a meromorphic continuation of $M_{w}^{(1)}(s)$ into $\Re s>-r$ for $r>k+1, r \in \mathbb{N}$, by the observation that

$$
M_{w}^{(1)}(s)=\int_{0}^{1}\left(\log \Phi_{w}(x)-q_{r-1}(x, w)\right) x^{s-1} d x+\sum_{\ell=k+1}^{r-1} \frac{b_{\ell}(w)}{s+\ell} .
$$

The right hand side of this equation is a meromorphic function in $\Re s>-r$ and therefore provides the meromorphic continuation of $M_{w}^{(1)}(s)$. Thus $M_{w}^{(1)}(s)$ has simple poles at $s=-\ell$ for $\ell \in\{k+1, k+2, \ldots\}$ with residues $b_{\ell}(w)$.

We proceed similarly for $M_{w}^{(2)}(s)$. This function is holomorphic for $\Re s<-\rho$. From Theorem 1 we know that the periodic function $F$ has a uniformly convergent Fourier series

$$
F(u)=\sum_{m \in \mathbb{Z}} f_{m} e^{2 \pi i m u} .
$$

From the fact that $F(u)$ is holomorphic for $|\Im u|<\beta \sigma$ (here and later we set $\left.\sigma=\frac{1}{\log \lambda}\right)$ we actually get that

$$
f_{m}=\mathcal{O}_{\varepsilon}(\exp (-2 \pi \sigma(\beta-\varepsilon)|m|)) \text { for all } \varepsilon>0 .
$$

Thus we can write

$$
\begin{array}{r}
M_{w}^{(2)}(s)=\int_{1}^{\infty}\left(\log \Phi_{w}(x)-q_{k}(x, w)-\sum_{m \in \mathbb{Z}} f_{m} x^{\rho+2 \pi i m \sigma}+\frac{\log a_{d}}{d-1}+\log (-w)\right) x^{s-1} d x \\
+\sum_{m \in \mathbb{Z}} \frac{f_{m}}{s+\rho+2 \pi i m \sigma}-\left(\frac{\log a_{d}}{d-1}+\log (-w)\right) \frac{1}{s},
\end{array}
$$

where the right hand side is a meromorphic function in $\mathbb{C}$ with simple poles at $s=$ $-\rho-2 \pi i m \sigma(m \in \mathbb{Z})$ with residue $f_{m}$ and at $s=0$ with residue $-\frac{\log a_{d}}{d-1}-\log (-w)$. This provides the meromorphic continuation of $M_{w}^{(2)}(s)$.

Thus we have proved

Lemma 3. The function $M_{w}(s)$ given by (5.3) admits a meromorphic continuation to the whole complex plane with simple poles at $s=-\ell$ for $\ell \in\{k+1, k+2, \ldots\}$ (with $k=\lfloor\rho\rfloor), s=0$, and $s=-\rho-2 \pi i m \sigma(m \in \mathbb{Z})$ with $\sigma=\frac{1}{\log \lambda}$. The residues at these poles are given by (the constants $b_{\ell}(w)$ are given by (5.2))

$$
\begin{aligned}
\operatorname{Res}_{s=-\ell} M_{w}(s) & =b_{\ell}(w) \quad \text { for } \ell \in\{k+1, k+2, \ldots\}, \\
\operatorname{Res}_{s=0} M_{w}(s) & =-\frac{\log a_{d}}{d-1}-\log (-w), \\
\operatorname{Res}_{s=-\rho-2 \pi i m \sigma} M_{w}(s) & =f_{m} \quad \text { for } m \in \mathbb{Z} .
\end{aligned}
$$

Under the conditions of Theorem 3, Corollary 4, or Corollary 5 infinitely many values $f_{m}$ are non-zero.

Similar arguments applied to the function

$$
M_{0}(s)=\int_{0}^{\infty}\left(\log \Phi(x)-\log x-\sum_{\ell=1}^{k} b_{\ell} x^{\ell}\right) x^{s-1} d x
$$


with $b_{\ell}$ given by

$$
\log \frac{\Phi(x)}{x}=\sum_{\ell=1}^{\infty} b_{\ell} x^{\ell}
$$

yield

Lemma 4. The function $M_{0}(s)$ given by (5.8) admits a meromorphic continuation to the whole complex plane with simple poles at $s=-\ell$ for $\ell \in\{k+1, k+2, \ldots\}$ (with $k=\lfloor\rho\rfloor)$, and $s=-\rho-2 \pi i m \sigma(m \in \mathbb{Z})$ with $\sigma=\frac{1}{\log \lambda}$, and a double pole at $s=0$. The residues at these poles (the principal part resp.) are given by (the constants $b_{\ell}$ are given by (5.9))

$$
\begin{aligned}
\operatorname{Res}_{s=-\ell} M_{0}(s) & =b_{\ell} \quad \text { for } \ell \in\{k+1, k+2, \ldots\}, \\
M_{0}(s) & =\frac{1}{s^{2}}-\frac{\log a_{d}}{d-1} \frac{1}{s}+\cdots, \\
\operatorname{Res}_{s=-\rho-2 \pi i m \sigma} M_{0}(s) & =f_{m} \quad \text { for } m \in \mathbb{Z} .
\end{aligned}
$$

Under the conditions of Theorem 3, Corollary 4, or Corollary 5 infinitely many values $f_{m}$ are non-zero.

In order to use the information derived for $M_{w}(s)$ for the meromorphic continuation of the Dirichlet series

$$
\zeta_{\Phi, w}(s)=\sum_{\substack{\Phi(-\mu)=w \\ \mu>0}} \mu^{-s}
$$

we derive an alternative expression for $M_{w}(s)$ based on (5.1):

$$
\begin{aligned}
& M_{w}(s)=\int_{0}^{\infty}\left(\log \Phi_{w}(x)-q_{k}(x, w)\right) x^{s-1} d x \\
& \quad=\sum_{\Phi_{w}(-\mu)=0} \int_{0}^{\infty}\left(\log \left(1+\frac{x}{\mu}\right)-\frac{x}{\mu}+\frac{x^{2}}{2 \mu^{2}}+\cdots+(-1)^{k} \frac{x^{k}}{k \mu^{k}}\right) x^{s-1} d x .
\end{aligned}
$$

Furthermore, the second integral in (5.12) can be evaluated as

$$
M_{w}(s)=\zeta_{\Phi, w}(-s) \frac{\pi}{s \sin \pi s}
$$

by the fact that for $-k-1<\Re s<-k$

$$
\int_{0}^{\infty}\left(\log (1+x)-x+\frac{x^{2}}{2}+\cdots+(-1)^{k} \frac{x^{k}}{k}\right) x^{s-1} d x=\frac{\pi}{s \sin \pi s}
$$

and the fact that the right hand side is the Mellin transform of a "harmonic sum" (cf. [28, 29]). Equation (5.13) provides us with the meromorphic continuation of $\zeta_{\Phi, w}(s)$. This is somewhat a reversion of the ideas used in [26].

Summing up, we have obtained

Theorem 7. Let $\Phi$ be the solution of the functional equation (3.1). Then the Dirichlet generating function $\zeta_{\Phi, w}(s)$ of the solutions of the equation $\Phi(-\mu)=w$ for $w<0$ given in (5.11) admits a meromorphic continuation to the whole complex plane. Under the conditions of Theorem 3, Corollary 4, or Corollary 5 there exist 
infinitely many simple poles at points of the form $s=\rho+2 \pi i m \sigma\left(\sigma=\frac{1}{\log \lambda}, m \in \mathbb{Z}\right)$. The following special values are known $\left(\rho=\frac{\log d}{\log \lambda}\right.$ and $f_{m}$ are given by (5.6)):

$$
\begin{aligned}
\underset{\rho+2 \pi i m \sigma}{\operatorname{Res}} \zeta_{\Phi, w}(s) & =-\frac{f_{m}}{\pi}(\rho+2 \pi i m \sigma) \sin \pi(\rho+2 \pi i m \sigma) \text { for } m \in \mathbb{Z}, \\
\zeta_{\Phi, w}(m) & =0 \quad \text { for } m<\rho, m \in \mathbb{Z}, \\
\zeta_{\Phi, w}^{\prime}(0) & =\frac{\log a_{d}}{d-1}+\log (-w), \\
\zeta_{\Phi, w}(m) & =(-1)^{m-1} m b_{m}(w) \quad \text { for } m \in \mathbb{N},
\end{aligned}
$$

where $b_{m}(w)$ is given by $(5.2)$.

Similar arguments applied to $\Phi(x) / x$ yield

Theorem 8. Let $\Phi$ be the solution of the functional equation (3.1). Then the Dirichlet generating function $\zeta_{\Phi, 0}(s)$ of the solutions of the equation $\Phi(-\mu)=0$ for $\mu>0$ given in (5.11) admits a meromorphic continuation to the whole complex plane. Under the conditions of Theorem 3, Corollary 4, or Corollary 5 there exist infinitely many simple poles at points of the form $s=\rho+2 \pi i m \sigma\left(\sigma=\frac{1}{\log \lambda}, m \in \mathbb{Z}\right)$. The following special values are known $\left(\rho=\frac{\log d}{\log \lambda}\right)$ :

$$
\begin{aligned}
\underset{\rho+2 \pi i m \sigma}{\operatorname{Res}} \zeta_{\Phi, 0}(s) & =-\frac{f_{m}}{\pi}(\rho+2 \pi i m \sigma) \sin \pi(\rho+2 \pi i m \sigma) \text { for } m \in \mathbb{Z}, \\
\zeta_{\Phi, 0}(m) & =0 \quad \text { for } m<\rho, m \in \mathbb{Z} \backslash\{0\}, \\
\zeta_{\Phi, 0}(0) & =1, \\
\zeta_{\Phi, 0}^{\prime}(0) & =\frac{\log a_{d}}{d-1}, \\
\zeta_{\Phi, 0}(m) & =(-1)^{m-1} m b_{m} \quad \text { for } m \in \mathbb{N},
\end{aligned}
$$

where $b_{m}$ is given by (5.9).

Remark 3. By Lemma 2 we have

$$
\zeta_{\Phi, w}(s)=\lim _{n \rightarrow \infty} \sum_{z \in p^{(-n)}(\{w\})}\left(\lambda^{n} z\right)^{-s},
$$

which is the function $\zeta_{p, w}(2 s)$ studied in $[41,42]$.

\section{Meromorphic Continuation of the Zeta FunCtion}

Up to now we have only considered the eigenvalues originating from $m=0$ in (2.1) for fixed $w \in A$. In general the multiplicity of eigenvalues for $m>0$ is given by a linear recurrent sequence $\beta_{m}(w)$. This sequence has a rational generating function

$$
B_{w}(x)=\sum_{m=0}^{\infty} \beta_{m}(w) x^{m}=\frac{P_{w}(x)}{Q_{w}(x)}
$$

with $P_{w}, Q_{w} \in \mathbb{Z}[x]$. Let $r_{w}$ denote the radius of convergence of $B_{w}$; then by Pringsheim's theorem $x=r_{w}$ is a pole of $B_{w}$. Let $k_{w}$ denote the order of this pole. 
Then the positivity of $\beta_{m}(w)$ implies that the coefficient $c_{k_{w}}(w)$ in the Laurentexpansion around $x=r_{w}$,

$$
B_{w}(x)=\frac{c_{k_{w}}(w)}{\left(1-\frac{x}{r_{w}}\right)^{k_{w}}}+\cdots,
$$

is positive.

Putting everything together, we can write

$$
\zeta_{\Delta}(s)=\sum_{-\Delta u=\mu u} \frac{1}{\mu^{s}}=\sum_{w \in A} \frac{P_{w}\left(\lambda^{-s}\right)}{Q_{w}\left(\lambda^{-s}\right)} \zeta_{\Phi, w}(s),
$$

which provides the meromorphic continuation of $\zeta_{\Delta}$ to the whole complex plane. If $\log _{\lambda} d<\frac{1}{2} d_{S}$, then all the functions $\zeta_{\Phi, w}(s)$ are holomorphic in $\Re s>\frac{1}{2} d_{S}-\varepsilon$ for some $\varepsilon>0$. Since $\zeta_{\Delta}(s)$ has a simple pole at $s=\frac{1}{2} d_{S}$ by the fact that $L(x) \asymp x^{\frac{1}{2} d_{S}}$, at least one of the rational functions $B_{w}(x)$ has to have a pole at $x=\lambda^{-\frac{1}{2} d_{S}}$. All poles of functions $B_{w}$ at $x=\lambda^{-\frac{1}{2} d_{S}}$ have to be simple. Let $W$ denote the set of those $w$ with $r_{w}=\lambda^{-\frac{1}{2} d_{S}}$. By our observation on the sign of $c_{1}(w)$ for $w \in W$ the Dirichlet series

$$
\eta(s)=-\sum_{w \in W} c_{1}(w) \zeta_{\Phi, w}(s)
$$

has positive coefficients. We apply [22, Theorem 9.5, p. 184] to the function $\eta(s)$ to see that $\eta\left(\frac{1}{2} d_{S}+i k \tau\right)=0$ cannot hold for fixed $\tau$ and all $k \in \mathbb{Z} \backslash\{0\}$. Thus the function

$$
\sum_{w \in W} B_{w}\left(\lambda^{-s}\right) \zeta_{\Phi, w}(s)
$$

has a simple pole at $s=\frac{1}{2} d_{S}$ and at least two non-real poles on the line $\Re s=\frac{1}{2} d_{S}$. Since the remaining summands in (6.1) have no poles on this line, these points remain poles of $\zeta_{\Delta}(s)$.

Summing up, we have proved:

Theorem 9. Let $G$ be a p.c.f. self-similar compact fractal, whose Laplace operator $\Delta$ admits spectral decimation in the sense of Definition 1 with a polynomial of degree $d$. Then the Dirichlet generating function of the eigenvalues of $\Delta$,

$$
\zeta_{\Delta}(s)=\sum_{-\Delta u=\mu u} \frac{1}{\mu^{s}},
$$

has a meromorphic continuation to the whole complex plane with poles contained in a finite union of sets $\left\{\rho_{k}+2 \pi i m \sigma \mid m \in \mathbb{Z}\right\}$, where $\sigma=\frac{1}{\log \lambda}$ and $\lambda$ is the parameter coming from spectral decimation. There is a simple pole at $s=\frac{1}{2} d_{S}\left(d_{S}\right.$ denoting the spectral dimension of $G)$. If $\log _{\lambda} d<\frac{1}{2} d_{S}$, then $\zeta_{\Delta}(s)$ has at least two non-real poles on the line $\Re s=\frac{1}{2} d_{S}$.

Remark 4 . The case of $G=[0,1]$ which gives the Riemann zeta function and has $\log _{\lambda} d=\frac{1}{2} d_{S}$ shows that the condition $\log _{\lambda} d<\frac{1}{2} d_{S}$ is needed for the last assertion.

Theorem 10. Let $G$ be a p.c.f. self-similar compact fractal, whose Laplace operator $\Delta$ admits spectral decimation in the sense of Definition 1 . Then the following are equivalent:

(1) $\zeta_{\Delta}(s)$ has at least two non-real poles in the set $\frac{1}{2} d_{S}+2 \pi i \sigma \mathbb{Z}\left(\sigma=\frac{1}{\log \lambda}\right)$; 
(2) the limit $\lim _{x \rightarrow \infty} x^{-\frac{1}{2} d_{S}} L(x)$ does not exist, where $L(x)$ denotes the eigenvalue counting function (1.1);

(3) the limit $\lim _{t \rightarrow 0+} P(t) t^{\frac{1}{2} d_{S}}$ does not exist, where $P(t)$ denotes the trace of the heat kernel (1.2).

Proof. " $(1) \Leftrightarrow(2)$ ": the general theory of Dirichlet series (cf. [11]) implies that $\zeta_{\Delta}(s)\left(\lambda^{s}-\lambda^{\frac{1}{2} d_{S}}\right)$ is of polynomial growth along vertical lines. Thus for some $k \in \mathbb{N}$ the last integral in the Mellin-Perron formula (cf. [39])

$$
\begin{aligned}
\sum_{\mu<x}\left(1-\frac{\mu}{x}\right)^{k}= & \frac{1}{2 \pi i} \int_{\frac{1}{2} d_{S}+1-i \infty}^{\frac{1}{2} d_{S}+1+i \infty} \zeta_{\Delta}(s) x^{s} \frac{d s}{s(s+1) \cdots(s+k)} \\
= & \sum_{\ell \in \mathbb{Z}} \operatorname{Res}_{s=\frac{1}{2} d_{S}+2 \pi i \ell \sigma} \zeta_{\Delta}(s) x^{s} \frac{1}{s(s+1) \cdots(s+k)} \\
& +\frac{1}{2 \pi i} \int_{\frac{1}{2} d_{S}-\varepsilon-i \infty}^{\frac{1}{2} d_{S}-\varepsilon+i \infty} \zeta_{\Delta}(s) x^{s} \frac{d s}{s(s+1) \cdots(s+k)}
\end{aligned}
$$

converges for some $\varepsilon>0$. The last integral is $\mathcal{O}\left(x^{\frac{1}{2} d_{S}-\varepsilon}\right)$. The series constitutes $x^{\frac{1}{2} d_{S}}$ times a Fourier series in $\log _{\lambda} x$, which is constant, if and only if there are no non-real poles on the line $\Re s=\frac{1}{2} d_{S}$. Since the left-hand side of the equation is an iterated integral of $L(x), x^{-\frac{1}{2} d_{S}} L(x)$ shows oscillations, if and only if the right-hand side does.

"(1) $\Leftrightarrow(3) "$ : again by Mellin inversion we get

$$
\begin{gathered}
P(t)=\frac{1}{2 \pi i} \int_{\frac{1}{2} d_{S}+1-i \infty}^{\frac{1}{2} d_{S}+1+i \infty} \zeta_{\Delta}(s) \Gamma(s) t^{-s} d s \\
\quad=\sum_{\ell \in \mathbb{Z}} \operatorname{Res}_{s=\frac{1}{2} d_{S}+2 \pi i \ell \sigma} \zeta_{\Delta}(s) \Gamma(s) t^{-s}+\frac{1}{2 \pi i} \int_{\frac{1}{2} d_{S}-\varepsilon-i \infty}^{\frac{1}{2} d_{S}-\varepsilon+i \infty} \zeta_{\Delta}(s) \Gamma(s) t^{-s} d s .
\end{gathered}
$$

Again the series is $t^{-\frac{1}{2} d_{S}}$ times a Fourier series in $\log _{\lambda} t$ and the last integral constitutes an error term $\mathcal{O}\left(t^{-\frac{1}{2} d_{S}+\varepsilon}\right)$. The same argument as above proves the presence of oscillations, if and only if $\zeta_{\Delta}$ has non-real poles on the line $\Re s=\frac{1}{2} d_{S}$.

Remark 5. In [17, p. 105] it is conjectured that $\lim _{x \rightarrow \infty} x^{-\frac{1}{2} d_{S}} L(x)$ does not exist for non-integer $d_{S}$ in the "lattice case". The non-existence of the limit has been confirmed in the case of existence of localised eigenfunctions (cf. [2, 24, 32]) and in the case of the existence of spectral gaps (cf. [38]).

Remark 6. In [19] it has been proved that the $n$-step transition probabilities $p_{n}(x, y)$ (the discrete analogue of the heat-kernel) on certain self-similar graphs satisfy an asymptotic relation

$$
p_{n}(x, y) \sim n^{-\frac{1}{2} d_{S}} F\left(\log _{\lambda} n\right)
$$


with a periodic function $F$. It is shown that $F$ is not constant if the Julia set of a related rational function is a Cantor set. In [43] it is proved for the same class of graphs that $F$ is not constant if $d_{S}$ is irrational.

Remark 7. Theorems 9 and 10 together confirm the conjecture (cf. [17, p. 105]) that the $\operatorname{limit} \lim _{x \rightarrow \infty} x^{-\frac{1}{2} d_{S}} L(x)$ does not exist for fractals admitting spectral decimation in the sense of Definition 1 with a polynomial of degree $d$ and $\log _{\lambda} d<$ $\frac{1}{2} d_{S}$.

\section{EXAMPLES}

Example 1. In $[9,33]$ the eigenvalues of the self-similar Dirichlet-Laplacian on the $K$-dimensional Sierpiński gasket have been identified. The $K$-dimensional gasket admits spectral decimation with the polynomial $p_{K}(x)=x(K+3+x)$. The eigenvalues of $\Delta$ are derived from the preiterates of the elements of the set $A=$ $\{-2,-(K+1),-(K+3)\}$ with multiplicities

$$
\begin{aligned}
\beta_{m}(-2) & = \begin{cases}1 & \text { for } m=1, \\
0 & \text { otherwise, }\end{cases} \\
\beta_{m}(-(K+1)) & =\frac{K-1}{2}(K+1)^{m-1}-\frac{K+1}{2} \text { for } m \geq 2, \\
\beta_{m}(-(K+3)) & =\frac{K-1}{2}(K+1)^{m-1}+\frac{K+1}{2} \text { for } m \geq 1 .
\end{aligned}
$$

This yields (with $\lambda=K+3$ )

$$
\begin{gathered}
\zeta_{\Delta}(s)=\lambda^{-s} \zeta_{\Phi,-2}(s)+\zeta_{\Phi,-(K+1)}(s) \sum_{m=2}^{\infty} \beta_{m}(-(K+1)) \lambda^{-m s} \\
+\zeta_{\Phi,-(K+3)}(s) \sum_{m=1}^{\infty} \beta_{m}(-(K+3)) \lambda^{-m s} \\
=\lambda^{-s} \zeta_{\Phi,-2}(s)+\left(\frac{K^{2}-1}{2\left(\lambda^{s}-(K+1)\right)}-\frac{K+1}{2\left(\lambda^{s}-1\right)}\right) \lambda^{-s} \zeta_{\Phi,-(K+1)}(s) \\
\quad+\left(\frac{K-1}{2\left(\lambda^{s}-(K+1)\right)}+\frac{K+1}{2\left(\lambda^{s}-1\right)}\right) \zeta_{\Phi,-(K+3)}(s) .
\end{gathered}
$$

We first observe that the poles of $\zeta_{\Phi, .}(s)$ at $s=\rho+2 \pi i m \sigma$, which are given by $\lambda^{s}=2$, cancel by the fact that the residues of $\zeta_{\Phi, w}$ at these points are independent of $w$ and the values of the rational factors sum up to 0. Furthermore, we observe

$$
\zeta_{\Phi,-(K+3)}(s)=\left(\lambda^{s}-1\right) \zeta_{\Phi, 0}(s)
$$

by the fact that $\Phi(z)=-(K+3)$, if and only if $\Phi(\lambda z)=0$ and $\Phi(z) \neq 0$. This implies that the poles at $s=2 \pi i m \sigma$ of the rational factor of the last summand in (7.1) are cancelled. Using Mellin transform, we see that the cancellation of poles on the line $\Re s=0$ is equivalent to the existence of the limit

$$
\lim _{x \rightarrow \infty} \frac{x^{\log _{\lambda}(-w)}}{\Phi(x)} \prod_{n=1}^{\infty}\left(1-\frac{1}{w} \Phi(x)\right) .
$$

We did some numerical computations, which indicate that there are oscillations for $w=-3$ and $K=2$. Nevertheless, the pole of the rational factor at $s=0$ is cancelled by the zero of $\zeta_{\Phi,-K-1}$. 
From this we derive, for instance,

$$
\begin{aligned}
\zeta_{\Delta}(0) & =\frac{K+1}{2}\left(1-2 \frac{\log (K+1)}{\log (K+3)}\right) \\
\zeta_{\Delta}^{\prime}(0) & =\log 2+\frac{2 K^{2}+3 K+1}{2 K} \log (K+1)-\frac{K^{2}+3 K-2}{4 K} \log (K+3) \\
& +\frac{K+1}{4 \log (K+3)} \zeta_{\Phi,-K-3}^{\prime \prime}(0)-\frac{K+1}{2 \log (K+3)} \zeta_{\Phi,-K-1}^{\prime \prime}(0), \\
\zeta_{\Delta}(1) & =\frac{K^{2}+3 K-1}{2(K+2)(K+3)} .
\end{aligned}
$$

Example 2. In $[40,41,42]$ the spectrum of the Neumann-Laplacian on the 2dimensional Sierpiński gasket has been studied. It has been shown that it admits spectral decimation with the polynomial $p(x)=x(5+x)$. The Neumann-eigenvalues are derived from the preiterates of the set $A=\{-3,-5\}$ with multiplicities

$$
\begin{aligned}
& \beta_{m}(-3)=\frac{3^{m-1}+3}{2} \text { for } m \geq 1, \\
& \beta_{m}(-5)=\frac{3^{m-1}-1}{2} \text { for } m \geq 2 .
\end{aligned}
$$

This gives

$$
\begin{aligned}
& \zeta_{\Delta}(s)=\zeta_{\Phi,-3}(s) \sum_{m=1}^{\infty} \beta_{m}(-3) 5^{-m s}+\zeta_{\Phi,-5}(s) \sum_{m=2}^{\infty} \beta_{m}(-5) 5^{-m s} \\
& =\left(\frac{1}{2\left(5^{s}-3\right)}+\frac{3}{2\left(5^{s}-1\right)}\right) \zeta_{\Phi,-3}(s)+\left(\frac{3}{2\left(5^{s}-3\right)}-\frac{1}{2\left(5^{s}-1\right)}\right) 5^{-s} \zeta_{\Phi,-5}(s),
\end{aligned}
$$

which is in accordance with [41, 42]. Notice that there is no pole at $s=0$, and the poles at the solutions of $5^{s}=1$ are cancelled in the second summand by the observation in Example 1. Numerical experiments seem to indicate that the poles of the first summand on the imaginary axis do not cancel with zeros of $\zeta_{\Phi,-3}$.

By the same arguments as in Example 1 the poles at the solutions of $5^{s}=2$ are cancelled. Furthermore, in the second summand the poles at the solutions of $5^{s}=1$ are cancelled by zeros of $\zeta_{\Phi,-5}$. The poles of the rational factors at $s=0$ cancel and we obtain

$$
\begin{aligned}
\zeta_{\Delta}(0) & =\frac{3}{2} \log _{5} 3-\frac{1}{2}, \\
\zeta_{\Delta}^{\prime}(0) & =\frac{3 \zeta_{\Phi,-3}^{\prime \prime}(0)-\zeta_{\Phi,-5}^{\prime \prime}(0)}{4 \log 5}-\log 3, \\
\zeta_{\Delta}(1) & =\frac{7}{30}, \\
\zeta_{\Delta}(2) & =\frac{1}{150} .
\end{aligned}
$$

The second derivatives $\zeta_{\Phi, w}^{\prime \prime}(0)$ can be computed numerically by the following observations. Around $s=0$ we can write $M_{w}(s)=-\frac{\log (-w)}{s}+H_{w}(s)$ with a function $H_{w}$ holomorphic around 0 , and by $(5.13)$ we have $2 H_{w}^{s}(0)=\zeta_{\Phi,-w}^{\prime \prime}(0)$. We 
have

$$
\begin{aligned}
& \left(1-2 \cdot 5^{s}\right) H_{w}(s)+d \frac{\log (-w)}{s}\left(5^{s}-1\right) \\
& \quad=\int_{0}^{1} \log \left(\frac{1-\frac{1}{w} \Phi(x)}{\left(1-\frac{1}{w} \Phi\left(\frac{x}{5}\right)\right)^{2}}\right) x^{s-1} d x+\int_{1}^{\infty} \log \left(\frac{1-\frac{1}{w} \Phi(x)}{-w\left(1-\frac{1}{w} \Phi\left(\frac{x}{5}\right)\right)^{2}}\right) x^{s-1} d x,
\end{aligned}
$$

and setting $s=0$,

$$
\begin{aligned}
H_{w}(0)=2 \log (-w) \log 5- & \int_{0}^{1} \log \left(\frac{1-\frac{1}{w} \Phi(x)}{\left(1-\frac{1}{w} \Phi\left(\frac{x}{5}\right)\right)^{2}}\right) \frac{d x}{x} \\
& -\int_{1}^{\infty} \log \left(\frac{1-\frac{1}{w} \Phi(x)}{-w\left(1-\frac{1}{w} \Phi\left(\frac{x}{5}\right)\right)^{2}}\right) \frac{d x}{x} .
\end{aligned}
$$

The first integral can be computed as the rapidly convergent series

$$
\int_{0}^{1} \log \left(\frac{1-\frac{1}{w} \Phi(x)}{\left(1-\frac{1}{w} \Phi\left(\frac{x}{5}\right)\right)^{2}}\right) \frac{d x}{x}=\sum_{n=1}^{\infty} \frac{b_{n}(w)}{n}\left(1-2 \cdot 5^{-n}\right) .
$$

The power series for $\log \left(1-\frac{1}{w} \Phi(z)\right)$ has radius of convergence the modulus of the smallest solution of $\Phi(z)=w$, which is much larger than 1 in the cases of interest. This gives exponential convergence.

For computing the second interval we observe that $\Phi(x) \geq \exp \left(0.9 x^{\rho}\right)$ for $x \geq 5$, which can be shown by discussing $\Phi$ on the finite interval $5 \leq x \leq 25$ and by extending the obvious inequality $\Phi(5 x) \geq \Phi(x)^{2}$. This together with an inequality for the logarithm yields (for $T \geq 25$ )

$$
0 \geq \int_{T}^{\infty} \log \left(\frac{1-\frac{1}{w} \Phi(x)}{-w\left(1-\frac{1}{w} \Phi\left(\frac{x}{5}\right)\right)^{2}}\right) \frac{d x}{x} \geq(2 w-1) \int_{T}^{\infty} \exp \left(-0.9 x^{\rho}\right) \frac{d x}{x}
$$

Thus the improper integral can be computed by truncation at a finite value and estimating the remainder integral as above.

Using these ideas we computed (using Mathematica)

$$
\begin{aligned}
H_{-3}(0) & =5.2399551500 \ldots, \\
H_{-5}(0) & =9.0660163789 \ldots, \\
\zeta_{\Delta}^{\prime}(0) & =0.9685221499 \ldots
\end{aligned}
$$

If there were no poles of $\zeta_{\Delta}$ on the line $\Re s=0, \exp \left(-\zeta_{\Delta}^{\prime}(0)\right)$ were the value of the regularised product of the eigenvalues (cf. [12]), or the Fredholm determinant of $\Delta$.

\section{ACKNOWLEDGMENT}

The second author is indebted to Michel Lapidus and Alexander Teplyaev for helpful discussions. The authors are grateful to an anonymous referee for valuable suggestions. 


\section{REFERENCES}

[1] M. T. Barlow, Diffusions on fractals, Lectures on probability theory and statistics (SaintFlour, 1995), Springer Verlag, Berlin, 1998, pp. 1-121. MR1668115 (2000a:60148)

[2] M. T. Barlow and J. Kigami, Localized eigenfunctions of the Laplacian on p.c.f. self-similar sets, J. London Math. Soc. (2) 56 (1997), 320-332. MR1489140 (99b:35162)

[3] M. T. Barlow and E. A. Perkins, Brownian motion on the Sierpiński gasket, Probab. Theory Relat. Fields 79 (1988), 543-623. MR966175 (89g:60241)

[4] A. F. Beardon, Iteration of rational functions, Graduate Texts in Mathematics, no. 132, Springer Verlag, 1991. MR1128089 (92j:30026)

[5] J. D. Biggins and N. H. Bingham, Near-constancy phenomena in branching processes, Math. Proc. Camb. Philos. Soc. 110 (1991), 545-558. MR1120488 (93d:60136)

[6] R. P. Boas, Jr., Entire functions, Academic Press Inc., New York, 1954. MR0068627 (16:914f)

[7] G. Doetsch, Handbuch der Laplace-Transformation. Band I: Theorie der LaplaceTransformation, Birkhäuser Verlag, Basel, 1971, Verbesserter Nachdruck der ersten Auflage 1950, Lehrbücher und Monographien aus dem Gebiete der exakten Wissenschaften. Mathematische Reihe, Band 14. MR0344807 (49:9546)

[8] S. Dubuc, Etude théorique et numérique de la fonction de Karlin-McGregor, J. Analyse Math. 42 (1982), 15-37. MR729400 (85g:30040)

[9] M. Fukushima and T. Shima, On a spectral analysis for the Sierpiński gasket, Potential Anal. 1 (1992), 1-35. MR1245223 (95b:31009)

[10] P. J. Grabner, Functional iterations and stopping times for Brownian motion on the Sierpinski gasket, Mathematika 44 (1997), 374-400. MR1600494 (99b:60128)

[11] G. H. Hardy and M. Riesz, The general theory of Dirichlet's series, Cambridge Tracts in Mathematics and Mathematical Physics, No. 18, Stechert-Hafner, Inc., New York, 1964. MR0185094 (32:2564)

[12] J. Jorgenson and S. Lang, Basic analysis of regularized series and products, Lecture Notes in Mathematics, vol. 1564, Springer-Verlag, Berlin, 1993. MR1284924 (95e:11094)

[13] S. Karlin and J. McGregor, Embeddability of discrete time simple branching processes into continuous time branching processes, Trans. Amer. Math. Soc. 132 (1968), 115-136. MR0222966 (36:6015)

[14] J. Kigami, A harmonic calculus for p.c.f. self-similar sets, Trans. Amer. Math. Soc. 335 (1993), 721-755. MR1076617 (93d:39008)

[15] J. Kigami, Distributions of localized eigenvalues of Laplacians on post critically finite selfsimilar sets, J. Funct. Anal. 156 (1998), 170-198. MR1632976 (99g:35096)

[16] _ Analysis on fractals, Cambridge Tracts in Mathematics, vol. 143, Cambridge University Press, Cambridge, 2001. MR1840042 (2002c:28015)

[17] J. Kigami and M. L. Lapidus, Weyl's problem for the spectral distribution of Laplacians on p.c.f. self-similar fractals, Comm. Math. Phys. 158 (1993), 93-125. MR1243717 (94m:58225)

[18] B. Krön, Green functions on self-similar graphs and bounds for the spectrum of the Laplacian, Ann. Inst. Fourier (Grenoble) 52 (2002), 1875-1900. MR1954327 (2003k:60180)

[19] B. Krön and E. Teufl, Asymptotics of the transition probabilities of the simple random walk on self-similar graphs, Trans. Amer. Math. Soc. 356 (2004), 393-414. MR2020038 (2004k:60130)

[20] M. Kuczma, On the Schröder equation, Rozprawy Mat. 34 (1963), 50. MR0173875 (30:4082)

[21] M. L. Lapidus, Analysis on fractals, Laplacians on self-similar sets, noncommutative geometry and spectral dimensions, Topol. Methods Nonlinear Anal. 4 (1994), 137-195. MR1321811 (96g:58196)

[22] M. L. Lapidus and M. van Frankenhuysen, Fractal geometry and number theory, Birkhäuser Boston Inc., Boston, MA, 2000, Complex dimensions of fractal strings and zeros of zeta functions. MR1726744 (2001b:11079)

[23] T. Lindstrøm, Brownian motion on nested fractals, Mem. Amer. Math. Soc., vol. 420, Amer. Math. Soc., 1990. MR988082 (90k:60157)

[24] L. Malozemov and A. Teplyaev, Pure point spectrum of the Laplacians on fractal graphs, J. Funct. Anal. 129 (1995), 390-405. MR1327184 (96e:60114)

[25] Self-similarity, operators and dynamics, Math. Phys. Anal. Geom. 6 (2003), 201218. MR1997913 (2004d:47012)

[26] H. Mellin, Die Dirichlet'schen Reihen, die zahlentheoretischen Funktionen und die unendlichen Produkte von endlichem Geschlecht, Acta Math. 28 (1903), 37-64. 
[27] S. Minakshisundaram and Å. Pleijel, Some properties of the eigenfunctions of the Laplaceoperator on Riemannian manifolds, Canadian J. Math. 1 (1949), 242-256. MR0031145 $(11: 108 \mathrm{~b})$

[28] F. Oberhettinger, Tables of Mellin transforms, Springer-Verlag, New York, 1974. MR0352890 (50:5376)

[29] R. B. Paris and D. Kaminski, Asymptotics and Mellin-Barnes integrals, Encyclopedia of Mathematics and its Applications, vol. 85, Cambridge University Press, Cambridge, 2001. MR1854469 (2002h:33001)

[30] R. Rammal and G. Toulouse, Random walks on fractal structures and percolation clusters, J. Physique Lettres 44 (1983), L13-L22.

[31] S. Rosenberg, The Laplacian on a Riemannian manifold, London Mathematical Society Student Texts, vol. 31, Cambridge University Press, Cambridge, 1997. MR1462892 (98k:58206)

[32] C. Sabot, Pure point spectrum for the Laplacian on unbounded nested fractals, J. Funct. Anal. 173 (2000), 497-524. MR1760624 (2001j:35216)

[33] T. Shima, On eigenvalue problems for the random walk on the Sierpiński pre-gaskets, Japan J. Appl. Ind. Math. 8 (1991), 127-141. MR1093832 (92g:60094)

[34] T. Shima, The eigenvalue problem for the Laplacian on the Sierpiński gasket, Asymptotic problems in probability theory: stochastic models and diffusions on fractals (Sanda/Kyoto, 1990), Pitman Res. Notes Math. Ser., vol. 283, Longman Sci. Tech., Harlow, 1993, pp. 279288. MR1354159 (96m:31015)

[35] _ On eigenvalue problems for Laplacians on p.c.f. self-similar sets, Japan J. Indust. Appl. Math. 13 (1996), 1-23. MR1377456 (97f:28028)

[36] R. S. Strichartz, Some properties of Laplacians on fractals, J. Funct. Anal. 164 (1999), 181-208. MR1695571 (2000f:35032)

[37] _ Fractafolds based on the Sierpinski gasket and their spectra, Trans. Amer. Math. Soc. 355 (2003), 4019-4043. MR1990573 (2004b:28013)

[38] L Laplacians on fractals with spectral gaps have nicer Fourier series, Math. Res. Lett. 12 (2005), 269-274. MR2150883 (2006e:28013)

[39] G. Tenenbaum, Introduction to analytic and probabilistic number theory, Cambridge Studies in Advanced Mathematics, no. 46, Cambridge University Press, 1995. MR1342300 (97e:11005b)

[40] A. Teplyaev, Spectral analysis on infinite Sierpiński gaskets, J. Funct. Anal. 159 (1998), 537-567. MR1658094 (99j:35153)

[41] _ Spectral zeta function of symmetric fractals, Fractal geometry and stochastics III (C. Bandt, U. Mosco, and M. Zähle, eds.), Progr. Probab., vol. 57, Birkhäuser, Basel, 2004, pp. 245-262. MR2087144 (2005h:28028)

[42] Spectral zeta functions of fractals and the complex dynamics of polynomials, available at http://arxiv.org/pdf/math.SP/0505546, 2005.

[43] E. Teufl, On the asymptotic behaviour of analytic solutions of linear iterative functional equations, Aequationes Math. (2006), to appear.

[44] G. Valiron, Fonctions analytiques, Presses Universitaires de France, Paris, 1954. MR0061658 $(15: 861 \mathrm{a})$

Department of Mathematics and Computer Science, Ben Gurion University of the Negev, Beer Sheva 84105, Israel

E-mail address: derfel@math.bgu.ac.il

Institut für Analysis und Computational Number Theory, Technische Universität Graz, Steyrergasse 30, 8010 Graz, Austria

E-mail address: peter.grabner@tugraz.at

Institut für Analysis und Scientific Computing, Technische Universität Wien, WiedNer Hauptstrasse 8-10, 1040 Wien, Austria

E-mail address: fvogl@osiris.tuwien.ac.at 